\title{
Atomic Scale Characterization of Strain Relaxation Behavior in LSFO/LSMO Superlattices
}

\author{
M. Gu${ }^{1}$, M. D. Biegalski ${ }^{2}$, H. M. Christen ${ }^{2}$, C. Song $^{3}$, C. R. Dearden ${ }^{1}$, N. T. Nguyen ${ }^{1}$, H. L. Vo ${ }^{1}$, Y.
} Takamura $^{1}$ and N. D. Browning ${ }^{1,4,5}$

${ }^{1}$ Department of Chemical Engineering and Materials Science, University of California-Davis, One Shields Avenue, Davis, CA 95616

${ }^{2}$ Center for Nanophase Materials Science, Oak Ridge National Laboratory, Oak Ridge, TN, 37831

${ }^{3}$ National Center for Electron Microscopy, MS 72-150, Lawrence Berkeley National Laboratory, Berkeley, CA 94720

${ }^{4}$ Department of Molecular \& Cellular Biology, University of California-Davis, One Shields Avenue, Davis, CA 95616

${ }^{5}$ Condensed Matter and Materials Division, Lawrence Livermore National Laboratory, Livermore, CA 94550

Superlattices consisting of the ferromagnetic metal $\mathrm{La}_{(1-\mathrm{x})} \mathrm{Sr}_{\mathrm{x}} \mathrm{MnO}_{3}(\mathrm{LSMO})$ and the antiferromagnetic insulator $\mathrm{La}_{(1-x)} \mathrm{Sr}_{\mathrm{x}} \mathrm{FeO}_{3}$ (LSFO) grown by pulsed laser deposition have the potential to be applied in solid oxide fuel cells as well as read heads in the hard drives [1,2]. The functional properties of these superlattices are closely related to the strain state and the uniformity of the structure. In this work, we identify the presence of defect structures in LSFO/LSMO superlattices with Sr doping, $\mathrm{x}=0.3$, by analyzing superlattices with different strain states.

The superlattices are characterized primarily utilizing high angle annular dark field (HAADF) Zcontrast imaging and bright field imaging in an aberration-corrected scanning transmission electron microscope (STEM). In strained [6LSFO][3LSMO]10 superlattices (6 unit cells of LSFO and 3 unit cells of LSMO repeated 10 times) grown on $\mathrm{SrTiO}_{3}$ (STO) substrates (Fig.1), no defect structures are seen and the film lattices is tetragonally distorted. In contrast, in strain-relaxed [6LSFO][3LSMO]10 superlattices, the film has nearly cubic structure and two types of stacking faults are detected. Fig2(a) shows a La-rich stacking fault which runs parallel to the growth direction and that is characterized by half unit cell shift of the lattice. The second defect region (Fig.2 (b)) possesses a reduced contrast difference between $\mathrm{A} / \mathrm{O}$ and $\mathrm{B} / \mathrm{O}$ atomic columns (see line profile in Fig.2(c)). Multi-slice image simulations and electron energy loss spectroscopy (EELS) confirmed that this contrast could result from a buried stacking fault, i.e. the same defect shown in Fig.2 (a) but lying in the plane of the image. For [6LSFO][6LSMO] superlattices with one-micron thickness (Fig.3), a high density of "nanoflowers" and cracks are observed by scanning electron microscopy (SEM). The $\mathrm{Z}$ contrast images show that these nanoflowers begin to form once a thickness reaches $\sim 250 \mathrm{~nm}$. EELS analysis reveal that these features are essentially a solid solution of $(\mathrm{LaSr})(\mathrm{FeMn}) \mathrm{O}_{3}$ (i.e. not showing discrete superlattice interfaces). The nearest atomic distance within the nanoflowers is 3.19 $\AA$, which corresponds well to the nearest atomic distance of the $<111>$ zone axis projection of a cubic perovskite structure with $3.905 \AA$ lattice parameter.

The accommodation of strain-relaxation in these superlattices is a possible explanation for the formation of these defects. La rich stacking faults can accommodate the tensile strain imposed by the STO substrates due to the larger size of the La ions, while the nanoflowers and cracks can result in the minimization of strain energy accumulated in the thicker samples. Our results show that these 
structures with low formation energy have faster growth rates, thus resulting in nanoflowers growing out of the superlattice film (Fig. 2(b)). [3]

\section{References}

[1] M. Izumi, et al, Materials Science and Engineering B84 (2001) 53

[2] Y. Takamura, et al., Phys. Rev. B, 80(2009) 180417(R)

[3] The research at UC Davis was supported by the NSF (contract No. DMR-747896) and DOE (contract No. DE-FG02-03ER46057). The work performed at NCEM is supported by the Office of Science, Office of Basic Energy Sciences of the U.S. Department of Energy under Contract No. DE-AC02-05CH1 1231. Part of this research was conducted at the Center for Nanophase Materials Sciences, which is sponsored at Oak Ridge National Laboratory by the Office of Basic Energy Sciences, U.S. Department of Energy.

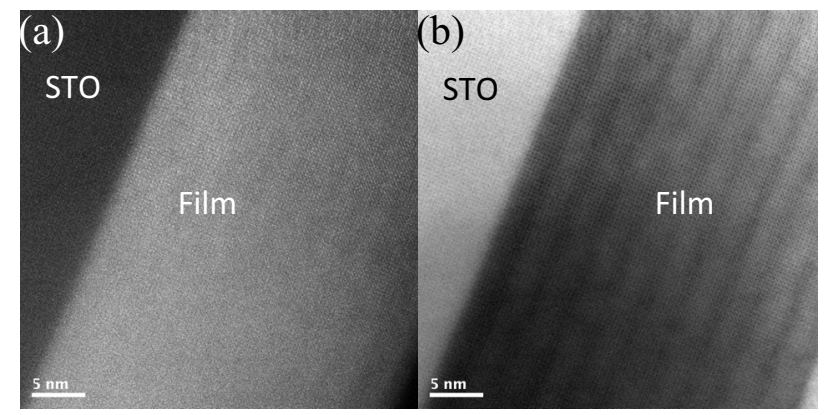

FIG. 1.(a) Z-contrast and (b) bright field image of a fully strained [6LSFO][3LSMO]10 superlattice.
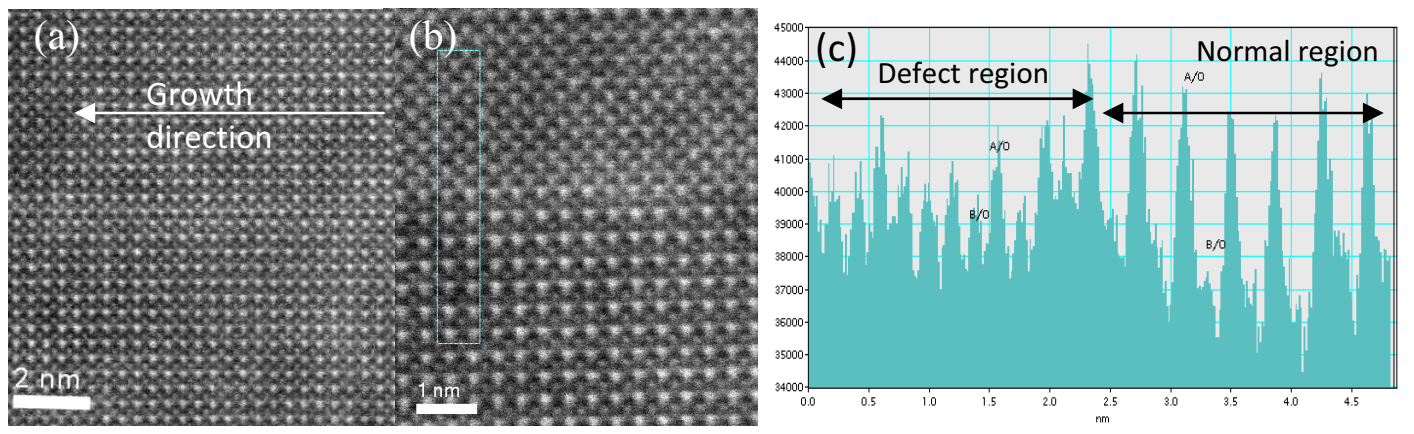

FIG. 2.(a) and (b) Atomic resolution Z-contrast images showing two types of stacking faults in the relaxed [6LSFO][3LSMO]10 superlattice. (c) Line profile of the blue box in (b).

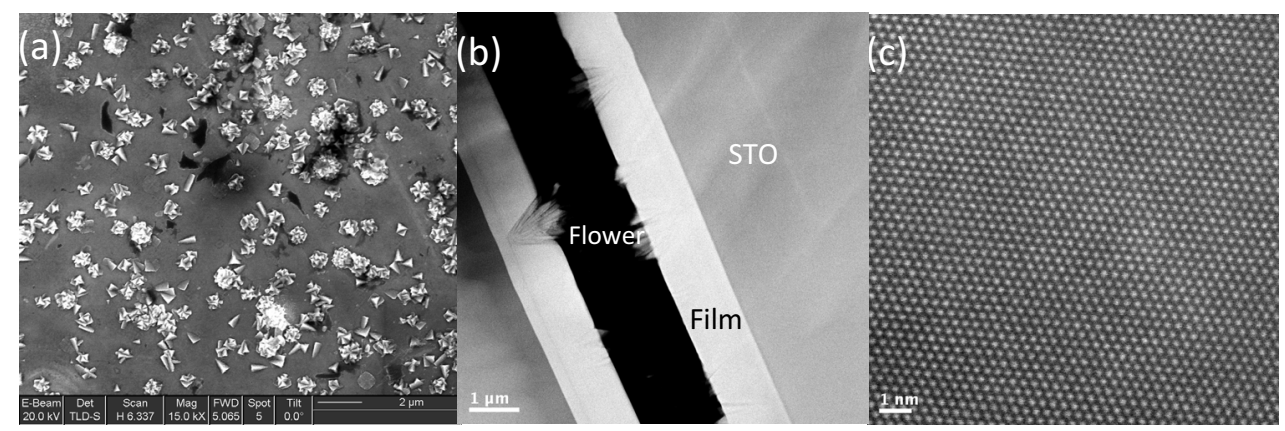

FIG. 3.(a) Planeview: SEM image, (b) cross section Z-contrast image, and (c) atomic resolution Zcontrast image along the $<111>$ zone axis projection of a nanoflower in a micron-thick [6LSFO][6LSMO] superlattices. 